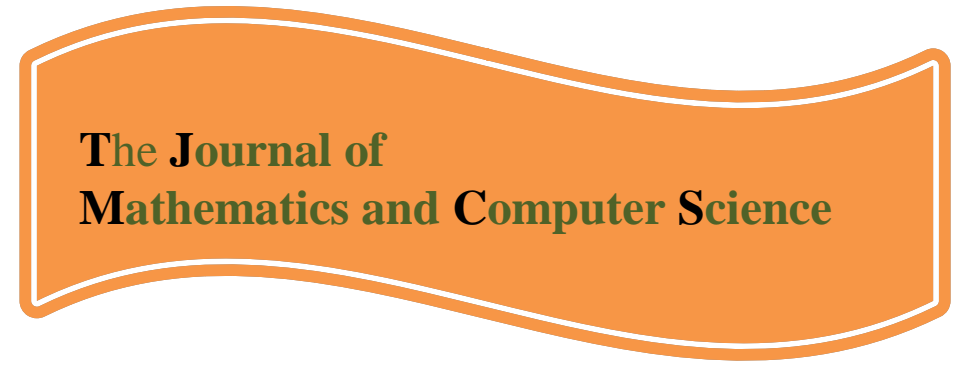

Available online at

\title{
http://www.TIMCS.com
}

\section{Application of Genetic Algorithm to Parameter Estimation in Liquid-Liquid Phase Equilibrium Modeling}

\author{
Mostafa Vatani \\ Department of Chemical Engineering, Faculty of Engineering, University of Kashan, \\ Kashan, P.0. Box 87317-51167, Iran \\ vatani_mostafa@grad.kashanu.ac.ir \\ Morteza Asghari \\ Department of Chemical Engineering, Faculty of Engineering, University of Kashan, \\ Kashan, P.0. Box 87317-51167, Iran \\ asghari@kashanu.ac.ir \\ Gholamreza Vakili-Nezhaad * \\ Department of Petroleum and Chemical Engineering, College of Engineering, Sultan Qaboos University, \\ P.O. Box 33, Muscat, Oman \\ Department of Chemical Engineering, Faculty of Engineering, University of Kashan, \\ Kashan, P.O. Box 87317-51167, Iran \\ vakili@squ.edu.om
}

Received: August 2012, Revised: November 2012

Online Publication: December 2012

* Corresponding author.

\begin{abstract}
Parameter estimation problems for liquid-liquid equilibrium data modeling are challenging due to the nonlinearity of thermodynamic models. In this work, the stochastic global optimization technique, namely Genetic Algorithm is applied to calculate the interaction and non randomness parameters of NRTL model. Forty ternary extraction systems containing benzene, hexane and different ionic liquids as solvent at various temperatures reported in literature are selected. The parameters of NRTL model are calculated and the global rmsd value of 0.0076 for 357 tie-lines shows that these parameters can be considered as the global parameters of NRTL model in the studied ternary systems. The global parameters are applied in all systems and rmsd values for them are reported. The results showed that Genetic Algorithm as a powerful and effective tool can be used to optimize highly nonlinear problems in phase equilibrium modeling.
\end{abstract}

Keywords: Genetic Algorithm, Parameter Estimation, NRTL model, Liquid-Liquid Equilibrium.

2010 Mathematics Subject Classification: Primary 54A40; Secondary 46S40. 


\section{Introduction}

Parameter estimation is very important in developing mathematical models of physical, chemical and biochemical processes. Parameter estimation in a (thermodynamic) model refers to determining the values of model parameters that best fit the model to the given experimental data set. The mathematical formulation involves the minimization of a suitable objective function subject to constraints arising from the model equations. For most of the thermodynamic models, the objective function and constraints are often highly nonlinear and non-convex. Hence, it is necessary to estimate the model parameters using global optimization methods because traditional optimization methods can only provide a local solution [1].

Modeling liquid phases in equilibrium with activity coefficient models frequently provides several local minima thus making the parameter estimation step a non trivial issue. In order to reduce the complexity involved, a global optimization algorithm may be required. For this purpose, the stochastic optimization algorithms have been proposed as interesting alternatives [2].

The focus of this work is finding the global interaction parameters of NRTL model for ternary extraction systems containing benzene, hexane and different ionic liquids at various temperatures. The method is based on Genetic Algorithm (GA) because it is example of some popular algorithms and can be used as the powerful and effective tool to optimize highly nonlinear problems in liquidliquid equilibrium modeling. Here the interaction and non randomness parameters of NRTL model are calculated for 40 ternary extraction systems. The results of NRTL model parameters are reported and the global rmsd value of 0.0076 for 357 tie-lines shows that these parameters can be considered as the global parameters of NRTL model in the studied ternary systems. The method can be extended to other fluid phase equilibria situations.

\section{Genetic Algorithm}

The Genetic Algorithm is an optimization and search technique based on the principles of genetics and natural selection. This algorithm allows a population composed of many individuals to evolve to an optimum solution under specified selection rules. It can be applied to solve a variety of engineering optimization problems that cannot be solved by traditional, derivative-based, optimization algorithms, including problems containing discontinuous, non-differentiable, or highly nonlinear objective functions.

GA differs from a derivative-based optimization algorithm in two main ways. Firstly, the derivativebased algorithm uses only one single point in each iteration, while GA explores the search space using multiple points. Secondly, the derivative-based algorithm generates new point by a deterministic computation.

In comparison, GA creates new population by a probabilistic computation. GA has been shown to solve linear and nonlinear problems by exploring all regions of the search space through selection, crossover, and mutation operations. A more complete discussion of GA, including extension and related topics, can be found in the Genetic Algorithm and Direct Search toolbox users' guide [3]. In the first step, an initial population is generated. Each individual is evaluated for fitness in the next step. Two individuals are chosen from the selection step. Individuals with high probability receive more probability to produce offspring. The offspring are then generated by combination of these selected individuals. In mutation step, random changes are applied to some individuals. This step aims to avoid a problem of overly fast convergence. The algorithm continues until termination criteria are reached. [4].

A procedure of GA is summarized in Figure. 1. 
M. Vatani, M. Asghari, G. Vakili-Nejhaad / TJ MCS Vol .5 No.1 (2012) 60-66

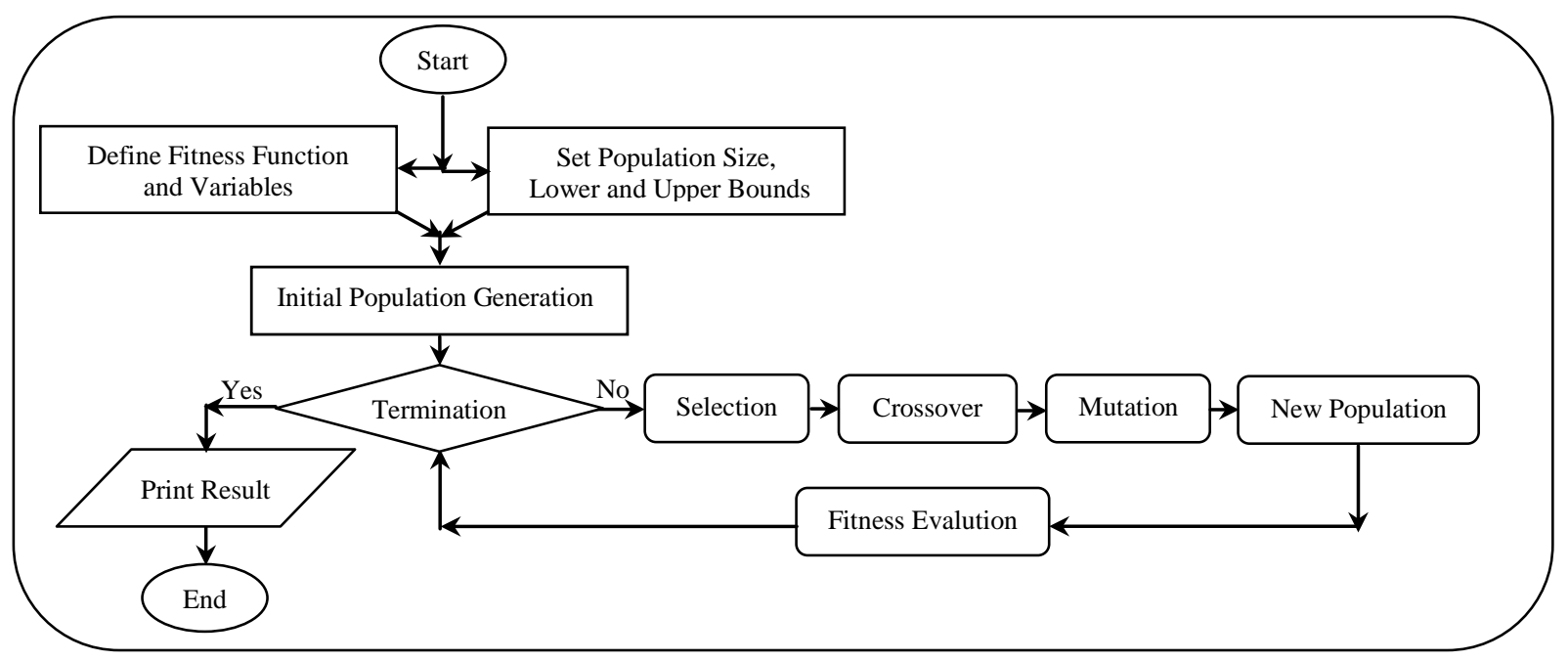

Figure 1. The flowchart of genetic algorithm toolbox

\section{NRTL Model}

The NRTL model [5] was used to correlate experimental liquid-liquid equilibrium data. The activity coefficient for NRTL model is given by following equation:

$$
\ln \gamma_{i}=\frac{\sum_{j=1}^{m} \tau_{j i} G_{j i} x_{j}}{\sum_{k=1}^{m} G_{k i} x_{k}}+\sum_{j=1}^{m} \frac{x_{j} G_{i j}}{\sum_{k=1}^{m} G_{k j} x_{k}}\left[\tau_{i j}-\frac{\sum_{k=1}^{m} x_{k} \tau_{k j} G_{k j}}{\sum_{k=1}^{m} G_{k j} x_{k}}\right]
$$

For this model, binary interaction $A_{i j}$ and and nonrandomness parameter $\alpha_{i j}$ have been defined as follows:

$$
\tau_{i j}=\frac{\Delta g_{i j}}{R T}=\frac{A_{i j}}{T} \text { and } G_{i j}=\exp \left(-\alpha_{i j} \tau_{i j}\right)
$$

where, in general

$$
A_{i i}=A_{j j}=0, A_{i j} \neq A_{j i} \text { and } \alpha_{i j}=\alpha_{j i} \text {. }
$$

\section{Parameter Estimation}

Parameter estimation is considered as a minimization of a proper objective function. The objective function $F_{o b j}$ which minimizes the deviation between the experimental and calculated mole fractions of the components can be expressed by:

$$
F_{o b j .}=\left[\sum_{i} \sum_{j} \sum_{k}\left(x_{i j k}^{\exp }-x_{i j k}^{c a l}\right)^{2}\right]
$$

The comparisons between experimental and calculated data can be made through root mean square deviations ( $\mathrm{rmsd}$ ) between the experimental and calculated composition of each component in both phases. This rmsd is given by: 
$r m s d=\left[\sum_{i} \sum_{j} \sum_{k}\left(x_{i j k}^{\mathrm{exp}}-x_{i j k}^{c a l}\right)^{2} / 6 M\right]^{1 / 2}$

where, $x$ is mole fraction; the subscripts $i, j$ and $k$ provide a designation for the component, phase, and tie-lines, respectively and the value $M$ designates the number of tie lines.

In the previous work [6], the procedure of LLE modeling is shown.

Table 1.

List of ternary systems, \{Benzene (1) +Hexane (2) +Ionic Liquid (3)\} along with Temperature, number of tie-lines and rmsd results for each system.

\begin{tabular}{|c|c|c|c|c|c|}
\hline Sys. no. & Ionic Liquid (3) & $\mathbf{T}(\mathbf{K})$ & Tie-line & rmsd & Ref. \\
\hline 1 & 1-ethylpyridinium ethylsulfate & 283.15 & 09 & 0.0068 & [7] \\
\hline 2 & 1-ethyl-3-methylpyridinium ethylsulphate & 283.15 & 10 & 0.0058 & {$[8]$} \\
\hline 3 & 1-ethyl-3-methylpyridinium ethylsulphate & 293.15 & 13 & 0.0056 & [8] \\
\hline 4 & 1-ethylpyridinium ethylsulfate & 298.15 & 09 & 0.0061 & [7] \\
\hline 5 & ethyl(2-hydroxyethyl)dimethylammonium bis\{(trifluomethyl)sulfonyl $\}$ imide & 298.15 & 09 & 0.0065 & [9] \\
\hline 6 & 1-ethyl-3-methylpyridinium ethylsulphate & 298.15 & 10 & 0.0052 & [8] \\
\hline 7 & 1-butyl-3-methylimidazolium bis\{(trifluoromethyl)sulfonyl\}-amide & 298.15 & 12 & 0.0092 & {$[10]$} \\
\hline 8 & 1-octhyl-3-methylimidazolium bis $\{$ (trifluoromethyl)sulfonyl $\}$-amide & 298.15 & 09 & 0.0149 & {$[10]$} \\
\hline 9 & 1-decyl-3-ethylimidazolium bis\{(trifluoromethyl)sulfonyl\}-amide & 298.15 & 11 & 0.0134 & {$[10]$} \\
\hline 10 & 1-dodecyl-3-ethylimidazolium bis\{(trifluoromethyl)sulfonyl $\}$-amide & 298.15 & 08 & 0.0114 & {$[10]$} \\
\hline 11 & 1-ethyl-3-methylimidazolium bis $\{$ (trifluoromethyl)sulfonyl $\}$-amide & 298.15 & 09 & 0.0079 & [11] \\
\hline 12 & 1-ethyl-3-methylimidazolium ethylsulfate & 298.15 & 08 & 0.0059 & [12] \\
\hline 13 & 1-ethyl-3-methylimidazolium ethylsulfate & 298.20 & 08 & 0.0047 & {$[13]$} \\
\hline 14 & 1-butyl-3-methylimidazolium methylsulfate & 298.20 & 08 & 0.0038 & [14] \\
\hline 15 & phosphonium based deep eutectic solvent (Salt:HBD molar ratios, 1:4)* & 300.15 & 08 & 0.0071 & {$[15]$} \\
\hline 16 & phosphonium based deep eutectic solvent (Salt:HBD molar ratios, 1:6) ${ }^{*}$ & 300.15 & 07 & 0.0068 & {$[15]$} \\
\hline 17 & phosphonium based deep eutectic solvent (Salt:HBD molar ratios, 1:6) ${ }^{*}$ & 300.15 & 07 & 0.0058 & {$[15]$} \\
\hline 18 & phosphonium based deep eutectic solvent (Salt:HBD molar ratios, 1:8) ${ }^{*}$ & 300.15 & 07 & 0.0057 & {$[15]$} \\
\hline 19 & 1-ethyl-3-methylpyridinium ethylsulphate & 303.15 & 10 & 0.0049 & [8] \\
\hline 20 & 3-methyl-N-butylpyridinium dicyanamide & 303.15 & 11 & 0.0062 & {$[16]$} \\
\hline 21 & 3-methyl-N-butylpyridinium tricyanomethanide & 303.20 & 10 & 0.0083 & [17] \\
\hline 22 & 3-methyl-N-butylpyridinium tetracyanoborate & 303.20 & 15 & 0.0089 & {$[18]$} \\
\hline 23 & phosphonium based deep eutectic solvent (Salt:HBD molar ratios, 1:4)* & 308.15 & 06 & 0.0078 & {$[15]$} \\
\hline 24 & phosphonium based deep eutectic solvent (Salt:HBD molar ratios, 1:6) & 308.15 & 07 & 0.0064 & {$[15]$} \\
\hline 25 & phosphonium based deep eutectic solvent (Salt:HBD molar ratios, 1:6) ${ }^{*}$ & 308.15 & 07 & 0.0057 & {$[15]$} \\
\hline 26 & 1-ethyl-3-methylimidazolium bis $\{$ (trifluoromethyl)sulfonyl $\}$-amide & 313.15 & 09 & 0.0080 & [11] \\
\hline 27 & 1-ethyl-3-methylimidazolium ethylsulfate & 313.20 & 08 & 0.0056 & {$[13]$} \\
\hline 28 & 1-butyl-3-methylimidazolium methylsulfate & 313.20 & 08 & 0.0041 & [14] \\
\hline 29 & 4-methyl-N-butylpyridinium tetrafluoroborate & 313.20 & 08 & 0.0052 & {$[19]$} \\
\hline 30 & phosphonium based deep eutectic solvent (Salt:HBD molar ratios, 1:4)* & 318.15 & 10 & 0.0080 & {$[15]$} \\
\hline 31 & phosphonium based deep eutectic solvent (Salt:HBD molar ratios, 1:4)* & 318.15 & 07 & 0.0057 & {$[15]$} \\
\hline 32 & phosphonium based deep eutectic solvent (Salt:HBD molar ratios, 1:6) & 318.15 & 07 & 0.0075 & {$[15]$} \\
\hline 33 & phosphonium based deep eutectic solvent (Salt:HBD molar ratios, 1:8) & 318.15 & 07 & 0.0068 & {$[15]$} \\
\hline 34 & phosphonium based deep eutectic solvent (Salt:HBD molar ratios, 1:8)* & 318.15 & 06 & 0.0100 & {$[15]$} \\
\hline 35 & 3-methyl-N-butylpyridinium dicyanamide & 328.15 & 10 & 0.0065 & {$[16]$} \\
\hline 36 & 1-ethyl-3-methylimidazolium ethylsulfate & 328.20 & 08 & 0.0067 & {$[13]$} \\
\hline 37 & 1-butyl-3-methylimidazolium methylsulfate & 328.20 & 08 & 0.0049 & [14] \\
\hline 38 & 3-methyl-N-butylpyridinium tetracyanoborate & 328.20 & 15 & 0.0094 & {$[18]$} \\
\hline 39 & 3-methyl-N-butylpyridinium tricyanomethanide & 328.20 & 10 & 0.0089 & {$[17]$} \\
\hline 40 & 4-methyl-N-butylpyridinium tetrafluoroborate & 333.20 & 08 & 0.0041 & {$[19]$} \\
\hline Global & & & 357 & 0.0076 & \\
\hline
\end{tabular}

${ }^{*}$ The mixing methyltriphenylphosphonium bromide as a salt with ethylene glycol as a hydrogen bond donor (HBD) 


\section{Results and Discussion}

To calculate the interaction parameters of NRTL model in ternary extraction ionic liquid systems, the stochastic global optimization method has been applied on 40 systems containing benzene, hexane and ionic liquids as listed in Table 1 . In this method, each new generation decreases the objective function.

In order to avoid numerical problems, the compositions of ionic liquids in the aliphatic rich phase were set to $10^{-6}$. GA uses random number generators algorithm which produces almost the same results at each runs. In order to find the lowest objective function, approximately 10 runs with random initial values of population, different random number generators, different operators and different lower and upper bounds were tested, resulted in different values of the objective function and rmsd and the lowest rmsd along with the corresponding parameters were selected as the final result and listed in Table 2.

The global rmsd value of 0.0076 for 357 tie-lines shows that these parameters can be considered as the global parameters of NRTL model in the studied ternary systems. The global parameters are applied in all of the systems and rmsd values for them are calculated and listed in Table 1 . The rmsd results for each system showed that these parameters are very satisfactory and can predict phase behaviors and tie-lines and can be applied for prediction of phase behavior of the systems containing benzene, hexane and different ionic liquids at different temperatures.

Figure 2 shows comparison between experimental and calculated tie-lines for the system Benzene (1) + Hexane (2) + [1-ethylpyridinium ethylsulfate] (3)\}. Similar figures could be plotted for all the systems in this work. It can be concluded that these parameters of NRTL model in systems of benzene, hexane and ionic liquids are able to predict the phase behavior of liquid-liquid equilibrium.

Table 2.

Global interaction parameters and rmsd in NRTL model for systems \{Benzene(1)+Hexane (2)+Ionic Liquids (3)\}.

\begin{tabular}{lllll}
\hline Components & $A_{i j}(\mathrm{~K})$ & $A_{j i}(\mathrm{~K})$ & $\alpha_{i j}$ & rmsd \\
\hline $1-2$ & 812.509 & 933.909 & 0.423 & \\
$1-3$ & 992.321 & 923.402 & 0.422 & 0.0076 \\
$2-3$ & 1398.470 & 1276.480 & 0.396 & \\
\hline
\end{tabular}

\section{Conclusions}

The ternary liquid-liquid equilibrium calculation was performed for 40 extraction systems containing benzene, hexane and ionic liquids based on flash calculation by NRTL activity coefficient model. A new approach based on the Genetic Algorithm had been successfully applied for calculation of fitting parameters in this model. The approach of this method is based on population, natural and stochastic selection in which no initial guesses are required. The parameters of NRTL model were calculated and the global rmsd value of 0.0076 for 357 tie-lines showed that these parameters can be considered as global parameters of NRTL model in the studied systems. The global parameters were applied in all of the 40 systems and rmsd values for them were calculated. The results for each system showed that these parameters were very satisfactory and can predict phase behaviors in systems of benzene, hexane and different ionic liquids at various temperatures. This approach can be applied to calculate the fitting parameters of other activity coefficient models in other equilibrium systems. 


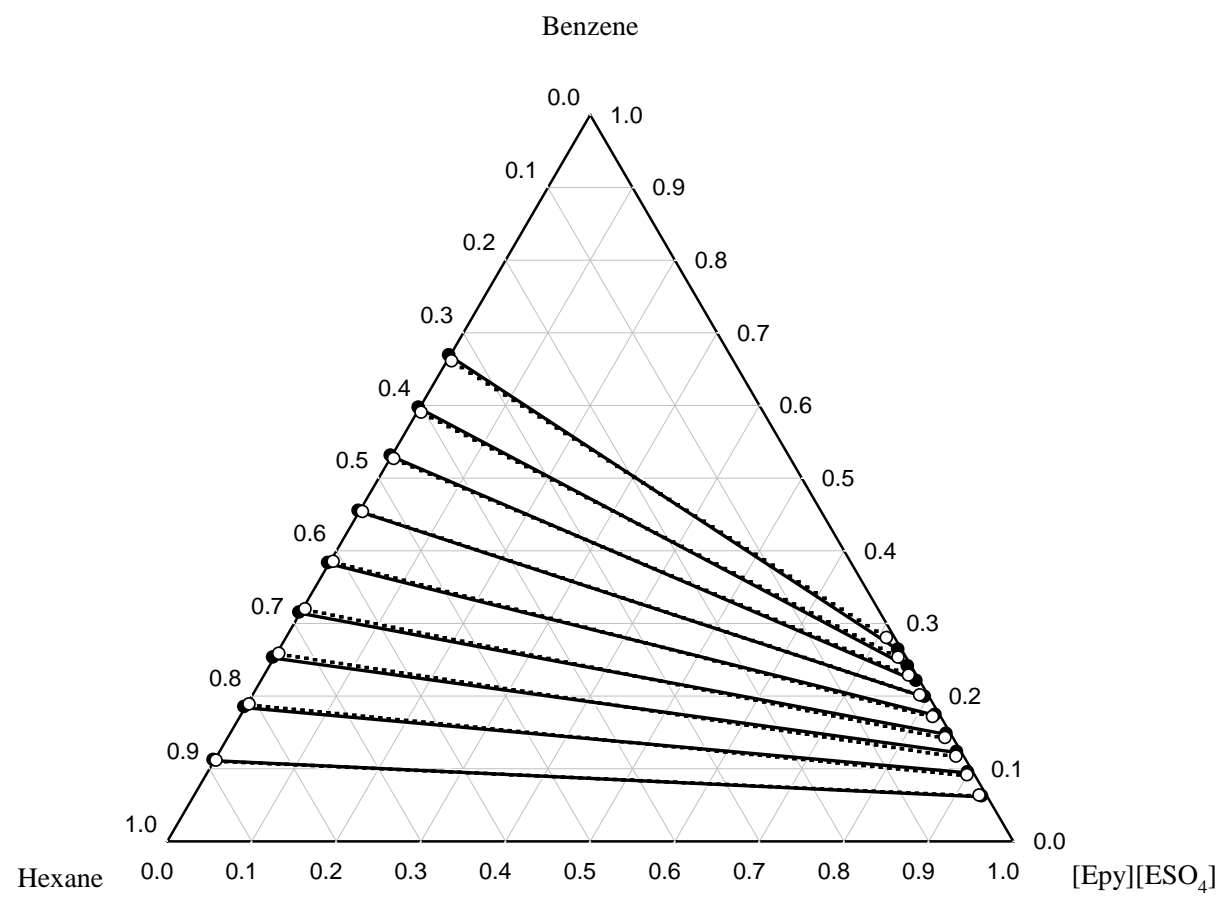

Figure 2. Tie-lines for System No. 1, Solid Lines and Full Points: Experimental tie-lines; Dashed Lines and Empty Points: Predicted tie-lines with NRTL Model.

\section{References}

[1] H. Zhang, D. Desfreed Kennedy, G. Pandu Rangaiah and A. Bonilla-Petriciolet, Novel bare-bones particle swarm optimization and its performance for modeling vapor-liquid equilibrium data, Fluid Phase Equilib., 301 (2011), 33-45.

[2] J.C. Ferrari, G. Nagatani, F.C. Corazza, J.V. Oliveira and M.L. Corazza, Application of stochastic algorithms for parameter estimation in the liquid-liquid phase equilibrium modeling, Fluid Phase Equilib., 280 (2009), 110-119.

[3] Genetic Algorithm and Direct Search Toolbox Users Guide, Copyright by the Math Works, Inc., 2004-2006.

[4] C. Preechakul and S. Kheawhom, Modified genetic algorithm with sampling techniques for chemical engineering optimization, J. Ind. Eng. Chem., 15 (2009), 110-118.

[5] H. Renon and J.M. Prausnitz, Local compositions in thermodynamic excess functions for liquid mixtures, AIChE Journal, 14 (1968), 135-144.

[6] M. Vatani, M. Asghari and G. Vakili-Nezhaad, Application of Genetic Algorithm to the calculation of parameters for NRTL and Two-Suffix Margules models in ternary extraction ionic liquid systems, J. Ind. Eng. Chem., 18 (2012), 1715-1720.

[7] E. Gómez, I. Domínguez, N. Calvar and A. Domínguez, Separation of benzene from alkanes by solvent extraction with 1-ethylpyridinium ethylsulfate ionic liquid, J. Chem. Thermodyn., 42 (2010), 1234-1239.

[8] E.J. González, N. Calvar, B. González and A. Domínguez, (Liquid + liquid) equilibria for ternary mixtures of (alkane + benzene + [EMpy] [ESO4]) at several temperatures and atmospheric pressure, J. Chem. Thermodyn., 41 (2009), 1215-1221. 
[9] U. Domanska, A. Pobudkowska and M. Krolikowski, Separation of aromatic hydrocarbons from alkanes using ammonium ionic liquid C2NTf2 at T = 298.15K, Fluid Phase Equilib., 259 (2007), 173-179.

[10] A. Arce, M.J. Earle, H. Rodriguez and K.R. Seddon, Separation of Benzene and Hexane by Solvent Extraction with 1-Alkyl-3-methylimidazolium Bis\{(trifluoromethyl)sulfonyl\}amide Ionic Liquids: Effect of the Alkyl-Substituent Length, J. Phys. Chem. B, 111 (2007), 4732-4736.

[11] A. Arce, M.J. Earle, H. Rodriguez and K.R. Seddon, Separation of aromatic hydrocarbons from alkanes using the ionic liquid 1-ethyl-3-methylimidazolium bis\{(trifluoromethyl) sulfonyl\}amide, Green Chem., 9 (2007), 70-74.

[12] E.J. Gonzalez, N. Calvar, E. Gomez and A. Domınguez, Separation of Benzene from Linear Alkanes (C6-C9) Using 1-Ethyl-3-Methylimidazolium Ethylsulfate at T = 298.15 K, J. Chem. Eng. Data, 55 (2010), 3422-3427.

[13] J. García, A. Fernández, J. Torrecilla, M. Oliet and F. Rodríguez, Liquid-liquid equilibria for \{hexane + benzene + 1-ethyl-3-methylimidazolium ethylsulfate $\}$ at $(298.2,313.2$ and 328.2)K, Fluid Phase Equilib., 282 (2009), 117-120.

[14] J. Garcia, A. Fernandez, J. Torrecilla, M. Oliet and F. Rodrıguez, Ternary Liquid-Liquid Equilibria Measurement for Hexane and Benzene with the Ionic Liquid 1-Butyl-3-methylimidazolium Methylsulfate at T = (298.2, 313.2, and 328.2) K, J. Chem. Eng. Data, 55 (2010), 258-261.

[15] M.A. Kareem, F.S. Mjalli, M.A. Hashim and I.M. AlNashef, Liquid-liquid equilibria for the ternary system (phosphonium based deep eutectic solvent-benzene-hexane) at different temperatures: A new solvent introduced, Fluid Phase Equilib., 314 (2012), 52- 59.

[16] A.R. Hansmeier, M. Jongmans, G.W. Meindersma and A.B. de Haan, LLE data for the ionic liquid 3-methyl-N-butyl pyridinium dicyanamide with several aromatic and aliphatic hydrocarbons, J. Chem. Thermodyn., 42 (2010), 484-490.

[17] G.W. Meindersma, T.V. Acker and A.B. de Haan, Physical properties of 3-methyl-Nbutylpyridinium tricyanomethanide and ternary LLE data with an aromatic and an aliphatic hydrocarbon at $\mathrm{T}=(303.2$ and 328.2) $\mathrm{K}$ and $\mathrm{p}=0.1 \mathrm{MPa}$, Fluid Phase Equilib., 307 (2011), 30- 38.

[18] G.W. Meindersma, B.T.J. Simons and A.B. de Haan, Physical properties of 3-methyl-Nbutylpyridinium tetracyanoborate and 1-butyl-1-methylpyrrolidinium tetracyanoborate and ternary LLE data of [3-mebupy] $\mathrm{B}(\mathrm{CN}) 4$ with an aromatic and an aliphatic hydrocarbon at $\mathrm{T}=303.2$ $\mathrm{K}$ and 328.2 K and p = 0.1 MPa, J. Chem. Thermodyn., 43 (2011), 1628-1640.

[19] G.W. Meindersma, A. Podt and A.B. de Haan, Ternary Liquid-Liquid Equilibria for Mixtures of an Aromatic + an Aliphatic Hydrocarbon + 4-Methyl-N-butylpyridinium Tetrafluoroborate, J. Chem. Eng. Data, 51 (2006), 1814-1819. 